\section{Anti-RNA Polymerase III Antibodies as a Risk Marker for Early Gastric Antral Vascular Ectasia (GAVE) in Systemic Sclerosis}

To the Editor:

We read with the greatest interest the recent article by Ingraham et al, on predictors of gastric antral vascular ectasia (GAVE) in systemic sclerosis $(\mathrm{SSc})^{1}$. In a series of 28 patients with GAVE, only 1 patient was positive for anti-topoisomerase I (topo I), whereas 1 patient had anti-RNA polymerase III (RNAP III) antibodies, and 4 (all with rapidly progressive diffuse cutaneous involvement) had a speckled antinuclear antibody (ANA) pattern. These 4 patients were not tested for anti-RNAP III antibodies, but the authors considered likely that the patients in this group would have anti-RNAP III because of their typical clinical appearance and the absence of anti-topo I. They also reported that, in their clinical practice, another 4 patients with diffuse cutaneous SSc and GAVE were found to be antiRNAP III positive. For these reasons, they raised the suggestion that antiRNAP III antibodies may act as predictors for early development of GAVE in SSc patients ${ }^{1}$.

We recently reported the clinical data of a series of anti-RNAP III positive SSc patients followed in our center ${ }^{2}$. Interestingly, 3 out of $18(16.7 \%)$ anti-RNAP III positive SSc patients had been diagnosed with GAVE ${ }^{2}$. This proportion is relatively high, considering that GAVE prevalence was estimated at $5.7 \%$ in a population of 264 consecutive patients with $\mathrm{SSc}^{3}$, and suggests a possible predictive role of anti-RNAP III antibodies, as reported by Ingraham, et al.

In order to verify the hypothesis that anti-RNAP III and anti-topo I antibodies are associated with different risk for early GAVE in SSc patients, we reevaluated the clinical charts of 453 consecutive patients with SSc and anti-RNAP III or anti-topo I antibodies. More than $99 \%$ of patients were Caucasian. The presence of GAVE was identified through endoscopic visual evidence in patients with SSc and unexplained iron deficiency anemia. Anti-topo I were detected by counterimmunoelectrophoresis and anti-RNAP III by ELISA.

Sixteen patients with isolated anti-RNAP III antibodies $(16 / 453 ; 3.5 \%)$ and 101 patients with anti-topo I $(101 / 453 ; 22.2 \%)$ were identified. Three additional patients with anti-RNAP III in combination with other antinuclear specificities (anti-NOR90, anti-topo I, anti-centromere, one case each) were excluded, due to the confounding effect of the double antinuclear specificity. Patients with anti-RNAP III antibodies had more rapid disease onset, defined as the interval from appearance of Raynaud's phenomenon to first symptom other than Raynaud's $(p=0.0013)$. They also had faster skin thickening in the first months after SSc onset $(p=0.0002)$, in comparison with anti-topo I positive SSc patients.
GAVE was diagnosed in 4/16 (25\%) SSc patients with anti-RNAP III antibodies, while no SSc patient with anti-topo I antibodies had GAVE $(0 / 101 ; p<0.0001)$. In two patients with GAVE the main sources of bleeding were the jejunum and the ileum, identified by video-capsule endoscopy. All 4 patients were diagnosed with GAVE within the first year of disease (mean $7 \mathrm{mo}$; SD: $1 \mathrm{mo}$ ), and they suffered from rapid progression of skin involvement.

Therefore, even considering the limitations of a retrospective study, our data suggest that SSc patients with rapid progression of cutaneous disease and anti-RNAP III antibodies are particularly at risk for the early development of GAVE. On the other hand, GAVE seems infrequent in patients with anti-topo I, as reported by others ${ }^{1,3}$. These observations lend further support to the role of SSc specific autoantibodies, such as anti-RNAP III and anti-topo I, which are differently associated with demographic, clinical, systemic, and survival features ${ }^{4}$. Thus, SSc autoantibodies can be very helpful in determining prognosis, as well as options for monitoring and treating patients with SSc. In particular, we agree with the suggestion by Ingraham, et al that, with commercial tests now available ${ }^{1}$, anti-RNAP III antibodies should be evaluated in all patients with SSc. Lastly, our experience suggests that video-capsule endoscopy may be a useful tool for SSc patients suspected to have early GAVE

ANGELA CERIBELLI, MD; ILARIA CAVAZZANA, MD; PAOLO AIRÒ, MD; FRANCO FRANCESCHINI, MD, Rheumatology Unit and Chair, Spedali Civili, Università degli Studi, Brescia, Italy.

\section{REFERENCES}

1. Ingraham KM, O’Brien MS, Shenin M, Derk CT, Steen V. Gastric antral vascular ectasia in systemic sclerosis: demographics and disease predictors. J Rheumatol 2010;37:603-7.

2. Cavazzana I, Ceribelli A, Airò P, Zingarelli S, Tincani A, Franceschini F. Anti-RNA polymerase III antibodies: a marker of systemic sclerosis with rapid onset and skin thickening progression. Autoimmun Rev 2009;8:580-4.

3. Marie I, Ducrotte P, Antonietti M, Herve S, Levesque H. Watermelon stomach in systemic sclerosis: its incidence and management. Aliment Pharmacol Ther 2008;28:412-21.

4. Steen VD. The many faces of scleroderma. Rheum Dis Clin North Am 2008;34:1-15.

5. Sidhu R, Sanders DS, McAlindon ME. Does capsule endoscopy recognise Gastric Antral Vascular Ectasia more frequently than conventional endoscopy? J Gastrointestin Liver Dis 2006;15:375-7.

J Rheumatol 2010;37:7; doi:10.3899/jrheum.100124 